\title{
Impact of the Dementia Care in Hospitals Program on acute hospital staff satisfaction
}

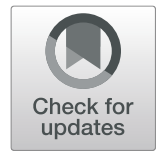

\author{
Margaret E. Murray ${ }^{1 *}$ (D, Anna Wong Shee ${ }^{1,2}$, Emma West ${ }^{3}$, Michelle Morvell ${ }^{2}$, Meredith Theobald ${ }^{2}$, \\ Vincent Versace ${ }^{1}$ and Mark Yates ${ }^{2}$
}

\begin{abstract}
Background: To evaluate the impact of the Dementia Care in Hospitals Program (DCHP) on clinical and non-clinical staff job satisfaction, level of confidence and comfort in caring for patients with cognitive impairment (Cl). Staff perceptions of how organisational support and hospital environment met the needs of patients with Cl were also assessed.

Methods: The DCHP was implemented across four acute hospital sites across Australia. Clinical and non-clinical staff received training on $\mathrm{Cl}$ screening and communication strategies for patients with $\mathrm{Cl}$. A staff satisfaction survey was administered pre- and post-implementation of the DCHP.

Results: One thousand seven hundred forty-eight staff received DCHP education and 1375 staff participated in the survey. Self-reported confidence and level of comfort in caring for patients with $\mathrm{Cl}$ significantly improved following implementation. Staff also reported increased job satisfaction and organisational support at all hospital sites.

Conclusions: The DCHP implementation within an acute hospital setting was found to show an improvement in staff confidence, comfort, and job satisfaction when caring for patients with $\mathrm{Cl}$. This study has significant implications for the improvement of care for patients with $\mathrm{Cl}$ as well as staff retention and job satisfaction. Further research is required to determine whether these improvements are sustained in the longer term.
\end{abstract}

Keywords: Cognitive impairment, Staff satisfaction, Education, Acute hospital

\section{Background}

Acute hospitals are increasingly being required to provide care for people with cognitive impairment $(\mathrm{CI})$. Over 83,000 people with CI are admitted to hospital each year in Australia [1]. Approaching 40\% of all patients over the age of 65 admitted to hospital have been shown to have a CI, yet acute hospitals are not equipped to meet the needs of these patients [2]. Cognitive impairment is associated with a person's decreased ability to think, formulate ideas, reason and remember, and includes dementia, delirium and other memory and thinking difficulties [3]. Patients with $\mathrm{CI}$ in acute hospitals are at a significantly increased risk of preventable complications such as falls, delirium, as well as adverse outcomes such as unexpected death, and unplanned entry into residential care [4]. The acute hospital environment can

\footnotetext{
* Correspondence: meg.murray@deakin.edu.au

'Deakin University, Geelong Australia, Deakin Rural Health, School of

Medicine, Warrnambool, VIC 3280, Australia

Full list of author information is available at the end of the article
}

often be unfamiliar and distressing to patients with $\mathrm{CI}$ [4]. The National Safety and Quality Health Service Standards emphasise the importance of a hospital culture that provides safe and high-quality care tailored to the needs of patients with CI [5].

Staff awareness of $\mathrm{CI}$, and the ability to identify and respond to patients with $\mathrm{CI}$ is fundamental to meeting the care and support needs of these patients and their carers [5]. Unlike other medical conditions, such as a fracture or a stroke, CI carries no visual stigmata, making it difficult to identify upon first contact by hospital staff. Furthermore, the diagnosis of $\mathrm{CI}$ within acute care can often be confounded by other illnesses, such as delirium [5]. Previous research has identified gaps in staff identification of patients with CI within the acute hospital environment [6]. There is a need to upskill acute hospital staff to identify patients with $\mathrm{CI}$, which is the crucial first step in the provision of appropriate care and minimising patients' risk of harm [5].

(c) The Author(s). 2019 Open Access This article is distributed under the terms of the Creative Commons Attribution 4.0 International License (http://creativecommons.org/licenses/by/4.0/), which permits unrestricted use, distribution, and reproduction in any medium, provided you give appropriate credit to the original author(s) and the source, provide a link to the Creative Commons license, and indicate if changes were made. The Creative Commons Public Domain Dedication waiver (http://creativecommons.org/publicdomain/zero/1.0/) applies to the data made available in this article, unless otherwise stated. 
Previous research has shown that when hospital staff lack the appropriate knowledge and skills for the management of patients with $\mathrm{CI}$, this results in low levels of confidence in their ability to care for these patients [7]. Staff require specific knowledge and skills to be able to respond appropriately to patients with CI. Best practice management and care of patients with $\mathrm{CI}$ includes: the identification and management of clinical risks, such as falls; targeted, individualised care; engagement with carers; appropriate management of behaviour; and delirium management and prevention strategies [8]. In addition, health professionals may feel uncomfortable discussing sensitive topics such as a person's cognitive status, and avoid the topic, resulting in less than optimal care [9]. Communication skills training is well recognised as key strategy for improving staff confidence when caring for patients with CI [10]. The growing numbers of patients with $\mathrm{CI}$ in acute hospitals highlights the need for staff in acute hospital settings to be trained to respond to patients with CI appropriately [4].

Creating a culture which provides high-quality care to patients with $\mathrm{CI}$, requires an all-of-hospital approach, where quality care is not solely the domain of nursing staff or health professionals. The ability of clinical and nonclinical staff to engage with patients with $\mathrm{CI}$ is critical for the provision of appropriate patient care and staff job satisfaction $[11,12]$. Hospital staff have frequently reported difficulty engaging with patients with $\mathrm{CI}$ and their families [13] and caring for patients with CI is frequently linked to low job satisfaction and burnout in care staff [14]. The provision of staff training and education have been identified as important strategies in enhancing the psychological well-being and job satisfaction of staff [15]. A clinical and non-clinical staff approach to education is essential for job satisfaction amongst staff working with patients with CI and for the delivery of high-quality, consistent care for these patients and their carers.

\section{Aim}

The aim of this study was to evaluate the impact of the Dementia Care in Hospitals Program (DCHP), a clinical and non-clinical staff education program designed to improve awareness of, and communication with patients with $\mathrm{CI}$, on staff confidence, comfort and job satisfaction in caring for patients with $\mathrm{CI}$.

\section{Methods}

\section{Hospital sites}

Four hospital sites located in South Australia, the Australian Capital Territory, Western Australia, and Tasmania, participants representing one regional and three metropolitan health services. All hospitals were tertiary hospitals with university affiliations, and the wards included were medical, surgical and aged care.

\section{Sample}

Clinical (nursing, medical, allied health, pathology, radiology,) and non-clinical staff (food and domestic services, porters/ orderlies, security, cleaning and hotel services, engineering, volunteers, administration, gardeners, and maintenance) that were employed at each of the four hospital sites, were invited to participate in the DCHP educational training.

\section{Intervention}

The intervention involved the implementation of the DCHP educational training program [16]. This program included:

1. Screening for $\mathrm{CI}$ of all patients aged 65 years and older or 50 years and over for an Aboriginal or Torres Strait Islander patients, using a validated screening tool [17].

2. Use of a Cognitive Impairment Identifier (CII) placed above the patient's bedside to alert staff of patients with CI (See Additional file 1).

3. Employment of a set of nine key communication strategies by all staff (clinical and non-clinical) who engaged with the patients (See Additional file 2).

Hospital training was based on a "train the trainer" approach [18] and delivered by the DCHP team. The hospitals then commenced an implementation of this training to all relevant clinical and non-clinical staff, with direct patient contact.

The DCHP was implemented at each of the four hospital sites over a nine-month period between: December 2015 to April 2017.

\section{Staff satisfaction survey}

Staff from each participating hospital site were invited to complete a staff satisfaction survey prior to and after implementation of the DCHP educational training program. The survey was developed in conjunction with Australian Institute for Primary Care and based on the literature and expert reviews. This survey consisted of five questions rated on a 5-point Likert scale, and three open-ended questions (See Additional files 3 and 4). It was designed to examine staff job satisfaction, as well as staff confidence and comfort in providing care for patients with CI. Staff perceptions of organisational support and how well equipped the hospital environment was to meet the needs of patients with $\mathrm{CI}$, were also assessed.

\section{Data collection}

Data collection commenced with the administration of the pre-intervention staff satisfaction 'baseline' survey. Commencement dates for the implementation of the 
DCHP were staged and the post-intervention survey was administered to staff at approximately 6 months postintervention, to allow for the embedding of the DCHP at each hospital site. Intervention surveys were administered at the beginning of the DCHP training sessions in the hospitals. Post-intervention surveys were collected on the wards following implementation of the DCHP.

\section{Data analysis}

Descriptive statistics including frequencies, means, and standard deviations were used to summarise the data. Independent $t$-tests were used to determine differences in pre- and post- DCHP education survey scores. All data were analysed using IBM SPSS 23.

\section{Results}

\section{Participant demographics}

A total of 1748 staff members $(67.5 \%)$ of the total workforce staff participated in the educational sessions. Nursing staff accounting for the highest proportion of staff trained $(n=909,52.0 \%)$, followed by non-clinical ( $n=315,18.0 \%)$, medical $(n=245,14.0 \%)$, allied health $(n=210,12.0 \%)$ and $69(3.9 \%)$ staff did not identify their role. Prior to the DCHP intervention, 159 (89.3\%) of the non-clinical staff had not previously received inservice or education on CI.

The numbers of staff that completed training and completed surveys pre- and post-implementation are shown in Fig. 1.

\section{Staff satisfaction survey}

\section{Staff attitude}

Prior to the intervention, the level of confidence reported amongst hospital staff was found to be highest for clinical staff, compared to non-clinical staff, with a significant improvement post-intervention $(p<0.001)$. The level of confidence reported amongst non-clinical staff also revealed a significant improvement post-intervention $(p<0.001)$. A similar significant finding was found for both clinical $(p<0.001)$ and non-clinical hospital staff $(p<0.001)$ who showed significant increases in level of comfort scores following the intervention phase. Job satisfaction also showed an overall increase across both clinical $(p<0.001)$ and non-clinical staff $(p<0.001)$ after the DCHP intervention (Table 1).
Following the intervention, staff confidence and comfort increased significantly across all four hospital sites. Post-intervention, job satisfaction was also found to have increased significantly for Site $1(p=0.001)$ and Site 2 $(p=0.002)$. Although Site 3 and Site 4 reported a similar increase in job satisfaction, these changes were not statistically significant (Table 2).

\section{Organisational support and hospital environment}

Prior to the intervention, staff-perceived level of organisational support for caring patients with $\mathrm{CI}$, was found to be highest for clinical staff. Clincial staff perceptions of organisation support for patients with CI significantly improved post-intervention $(p<0.001)$ (Table 3). In contrast, staff perceptions of how well equipped the hospital environment was to meet the needs of patients with CI, was higher for non-clinical staff.

Post-intervention, staff perceptions of organisational support significantly increased for all of the four hospital sites (Table 4). Similarly, staff perceptions of how well equipped the hospital environment was in meeting the needs of patients with CI significantly increased postintervention at Site $4(p<0.001)$.

\section{Staff engagement with patients with cognitive impairment and their carers}

Clinical staff were more likely to report a problem or difficulty in working with patients and/or their carers compared to non-clinical staff. Prior to the DCHP intervention, $(n=722,89.6 \%)$ clinical staff reported a problem or difficulty in working with patients with $\mathrm{CI}$, and approximately two-thirds reported a problem or difficulty in working with their carers and/or family. Following the intervention, clinical staff showed a statistically significant decrease in the percentage staff experiencing difficulties working with patients with $\mathrm{CI}$ and their carers (Table 5).

\section{Discussion}

This study is the first evaluation of the impact of the clinical and non-clinical staff education program on staff confidence, comfort and job satisfaction in caring for patients with CI. Overall, staff reported an increased ability to communicate with and respond appropriately towards patients with $\mathrm{CI}$, following the implementation of the DCHP educational training program. Importantly, staff

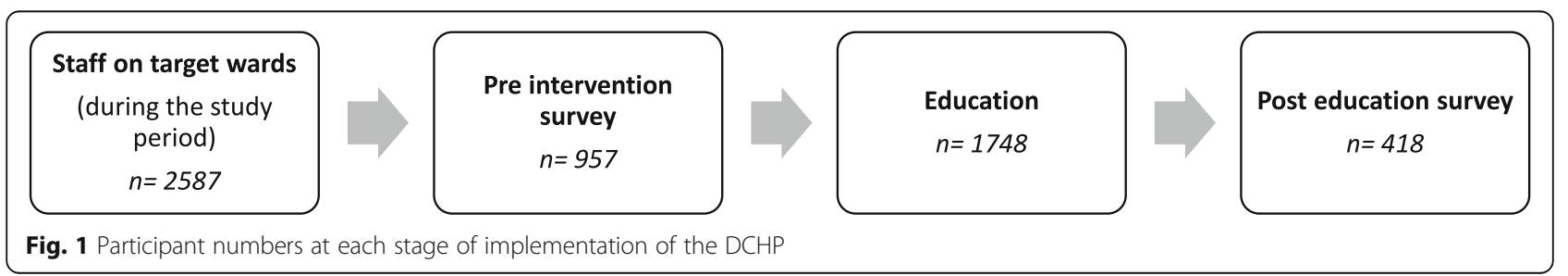


Table 1 Staff self-rated confidence, comfort and job satisfaction: pre and post-intervention

\begin{tabular}{|c|c|c|c|c|c|c|}
\hline \multirow{2}{*}{$\begin{array}{l}\text { Self-rated } \\
\text { measures }\end{array}$} & \multicolumn{2}{|c|}{ Pre-Intervention } & \multicolumn{2}{|c|}{ Post-Intervention } & \multirow[t]{2}{*}{$\mathrm{t}$} & \multirow[t]{2}{*}{$P$} \\
\hline & $n$ & Mean (SD) & $n$ & Mean (SD) & & \\
\hline \multicolumn{7}{|l|}{ Confidence } \\
\hline Clinical staff & 725 & $3.23(0.73)$ & 318 & $3.52(0.80)$ & -5.66 & $<0.001^{* *}$ \\
\hline Non clinical staff & 150 & $2.79(0.93)$ & 26 & 3.69 (0.79) & -4.65 & $<0.001^{* *}$ \\
\hline All staff & 954 & $3.15(0.79)$ & 418 & $3.52(0.79)$ & -7.98 & $<0.001^{* *}$ \\
\hline \multicolumn{7}{|l|}{ Comfort } \\
\hline Clinical staff & 724 & $3.24(0.80)$ & 317 & $3.55(0.80)$ & -5.71 & $<0.001^{* *}$ \\
\hline Non clinical staff & 150 & $2.92(0.87)$ & 26 & $3.54(0.81)$ & -3.38 & $<0.001^{* *}$ \\
\hline All staff & 953 & $3.18(0.82)$ & 417 & $3.53(0.81)$ & -7.33 & $<0.001^{* *}$ \\
\hline \multicolumn{7}{|l|}{ Job Satisfaction } \\
\hline Clinical staff & 719 & $2.88(0.77)$ & 316 & $3.08(0.79)$ & -3.75 & $<0.001^{* *}$ \\
\hline Non clinical staff & 139 & $2.92(0.80)$ & 26 & $3.54(0.86)$ & -3.57 & $<0.001^{* *}$ \\
\hline All staff & 936 & $2.88(0.79)$ & 415 & $3.11(0.82)$ & -4.70 & $<0.001^{* *}$ \\
\hline
\end{tabular}

** Significant at the 0.001 level

reported improved job satisfaction when working with patients with $\mathrm{CI}$ and their carers. Our findings show that the DCHP is effective in improving staff confidence, comfort, and job satisfaction when working with patients with CI.

Hospital staff require the necessary skills and knowledge to provide quality care to patients with $\mathrm{CI}$, including how to identify, communicate and respond appropriately to patients with CI [5]. To the best of our knowledge, this study is the first to include non-clinical hospital staff, as these staff play an important role in the daily provision of care

Table 2 Staff self-rated confidence, comfort and job satisfaction at each hospital site: pre and post-intervention

\begin{tabular}{|c|c|c|c|c|c|c|}
\hline \multirow{2}{*}{$\begin{array}{l}\text { Self-rated } \\
\text { measures }\end{array}$} & \multicolumn{2}{|c|}{ Pre-Intervention } & \multicolumn{2}{|c|}{ Post-Intervention } & \multirow[t]{2}{*}{$\mathrm{t}$} & \multirow[t]{2}{*}{ P } \\
\hline & $\mathrm{n}$ & Mean (SD) & $n$ & Mean (SD) & & \\
\hline \multicolumn{7}{|c|}{ Confidence } \\
\hline Site 1 & 234 & $3.24(0.74)$ & 40 & $3.73(0.78)$ & -3.75 & $<.001^{* *}$ \\
\hline Site 2 & 305 & $2.99(0.86)$ & 160 & $3.48(0.78)$ & -5.93 & $<.001^{* *}$ \\
\hline Site 3 & 237 & $3.24(0.78)$ & 137 & $3.56(0.74)$ & -3.98 & $<.001^{* *}$ \\
\hline Site 4 & 178 & $3.19(0.70)$ & 81 & $3.44(0.88)$ & -2.48 & $0.02^{*}$ \\
\hline \multicolumn{7}{|l|}{ Comfort } \\
\hline Site 1 & 234 & $3.15(0.75)$ & 40 & $3.75(0.78)$ & -4.63 & $<.001^{* *}$ \\
\hline Site 2 & 305 & $3.09(0.87)$ & 160 & $3.44(0.82)$ & -4.15 & $<.001^{* *}$ \\
\hline Site 3 & 237 & $3.28(0.81)$ & 136 & $3.60(0.75)$ & -3.83 & $<.001^{* *}$ \\
\hline Site 4 & 177 & $3.21(0.83)$ & 81 & $3.47(0.87)$ & -2.25 & $0.03^{*}$ \\
\hline \multicolumn{7}{|c|}{ Job Satisfaction } \\
\hline Site 1 & 232 & $2.83(0.77)$ & 40 & $3.30(0.79)$ & -3.52 & $<.001^{* *}$ \\
\hline Site 2 & 294 & $2.84(0.79)$ & 157 & $3.10(0.83)$ & -3.16 & $0.002^{*}$ \\
\hline Site 3 & 234 & $3.00(0.82)$ & 137 & $3.12(0.77)$ & -1.35 & 0.18 \\
\hline Site 4 & 176 & $2.87(0.75)$ & 81 & $3.01(0.89)$ & -1.34 & 0.18 \\
\hline
\end{tabular}

*Significant at the 0.05 level

** Significant at the 0.001 level for patients with $\mathrm{CI}$, and also require specialised CI training. Our findings show that the self-reported staff confidence and level of comfort in caring for patients with CI improved, following the implementation of the DCHP, for both clinical and non-clinical staff. These findings are consistent with a previous UK study conducted by Hughes et al. [19], which examined the knowledge and confidence of nursing and care assistant staff, in caring for people with dementia. It was found that although staff possessed adequate knowledge of dementia, staff confidence was lower, and could be positively influenced by additional training [19]. Similar to these findings, Sampson et al. [20], implemented a 'train-the-trainer' model across eight acute hospitals in the UK, and found that both clinical and nonclinical staff appeared more confident at engaging and responding to non-verbal cues in people with dementia. Utilising a clinical and non-clinical staff approach to education, is an important factor to increasing confidence for both clinical and non-clinical hospital staff, when working with patients with $\mathrm{CI}$ and their carers.

Staff-perceived difficulties when working with patients with $\mathrm{CI}$, such as challenges communicating with patients, a lack of staff skills and understanding of CI, disruptive behaviours, and inadequate hospital resources, have been widely reported [13]. In this study, clinical staff reported experiencing less difficulty working with patients with $\mathrm{CI}$ and their carers following implementation of the DCHP. This is consistent with the findings of Surr et al. [15], who evaluated a specialist training programme for acute clinical hospital staff, in regards to staff attitudes towards the provision of care for people with dementia. That study showed that following the training programme, a significant positive change in staff attitudes was reported. In contrast, this current study found the perceived difficulty working with patients with 
Table 3 Staff self-rated organisational support and hospital environment: pre and post-intervention

\begin{tabular}{|c|c|c|c|c|c|c|}
\hline \multirow{2}{*}{$\begin{array}{l}\text { Self-rated } \\
\text { measures }\end{array}$} & \multicolumn{2}{|c|}{ Pre-Intervention } & \multicolumn{2}{|c|}{ Post-Intervention } & \multirow[t]{2}{*}{$\mathrm{t}$} & \multirow[t]{2}{*}{$P$} \\
\hline & $n$ & Mean (SD) & $n$ & Mean (SD) & & \\
\hline \multicolumn{7}{|c|}{ Organisational Support } \\
\hline Clinical staff & 722 & $2.86(0.81)$ & 318 & $3.20(0.90)$ & -5.99 & $<0.001^{* *}$ \\
\hline Non clinical staff & 144 & $2.58(0.96)$ & 26 & $3.62(0.85)$ & -5.11 & $<0.001^{* *}$ \\
\hline All staff & 944 & $2.80(0.85)$ & 418 & $3.20(0.91)$ & -7.84 & $<0.001^{* *}$ \\
\hline \multicolumn{7}{|l|}{ Hospital Environment } \\
\hline Clinical staff & 720 & $2.55(0.81)$ & 317 & $2.69(0.88)$ & -2.53 & $0.010^{*}$ \\
\hline Non clinical staff & 142 & $2.80(0.96)$ & 26 & $3.46(0.76)$ & -3.33 & $0.001^{* *}$ \\
\hline All staff & 939 & $2.60(0.86)$ & 415 & $2.74(0.90)$ & -2.75 & $0.010^{*}$ \\
\hline
\end{tabular}

*Significant at the 0.05 level

** Significant at the 0.001 level

CI to have increased amongst non-clinical staff, following DCHP implementation. The differences in findings between clinical and non-clinical staff may result from the varying degree of contact these two staff groups have with patients and their carers. Consistent with a higher level of patient contact amongst clinical staff, changes in their experiences with patients with $\mathrm{CI}$ and their carers, may also be easier to identify. Increasing staff skills in identifying and responding to patients with $\mathrm{CI}$ allows staff to deliver appropriate care and engage with these patients and their carers.

The ability of staff to engage with patients with $\mathrm{CI}$ and their carers can directly impact on staff job satisfaction [13]. Our study shows that overall, the DCHP improved staff perceived level of job satisfaction in working with patients with CI. These findings for clinical staff are consistent with previous research, where the level of knowledge in regards to $\mathrm{CI}$, contributed significantly to job satisfaction of hospital employees [15]. That study showed that a specialist training programme significantly

Table 4 Staff self-rated organisational support and hospital environment at each hospital site: pre and post-intervention

\begin{tabular}{|c|c|c|c|c|c|c|}
\hline \multirow{2}{*}{$\begin{array}{l}\text { Self-rated } \\
\text { measures }\end{array}$} & \multicolumn{2}{|c|}{ Pre-Intervention } & \multicolumn{2}{|c|}{ Post-Intervention } & \multirow[t]{2}{*}{$\mathrm{t}$} & \multirow[t]{2}{*}{$P$} \\
\hline & $n$ & Mean (SD) & $\mathrm{n}$ & Mean (SD) & & \\
\hline \multicolumn{7}{|c|}{ Organisational Support } \\
\hline Site 1 & 233 & $2.70(0.77)$ & 40 & $3.03(0.97)$ & -2.33 & $0.020^{*}$ \\
\hline Site 2 & 301 & $2.69(0.84)$ & 160 & $2.97(0.95)$ & -3.20 & $0.001^{* *}$ \\
\hline Site 3 & 233 & $2.97(0.96)$ & 137 & $3.42(0.78)$ & -4.57 & $<0.001^{* *}$ \\
\hline Site 4 & 177 & $2.88(0.79)$ & 81 & $3.38(0.90)$ & -4.58 & $<0.001^{* *}$ \\
\hline \multicolumn{7}{|c|}{ Hospital Environment } \\
\hline Site 1 & 234 & $2.53(0.80)$ & 40 & $2.53(0.93)$ & 0.01 & 0.996 \\
\hline Site 2 & 295 & $2.52(0.86)$ & 157 & $2.66(0.94)$ & -1.56 & 0.120 \\
\hline Site 3 & 233 & $2.95(0.87)$ & 137 & $2.88(0.83)$ & 0.79 & 0.430 \\
\hline Site 4 & 177 & $2.37(0.77)$ & 81 & $2.78(0.91)$ & -3.76 & $<0.001^{* *}$ \\
\hline
\end{tabular}

*Significant at the 0.05 level

** Significant at the 0.001 level improved job satisfaction for clinical staff working with people with dementia in an acute hospital. While all of the hospital sites showed an improvement in job satisfaction, two of the sites did not demonstrate significant improvements. This may have been the result of variations in the implementation of the DCHP between hospital sites. Hospital sites were provided with the initial training by the DCHP team, however the implementation of the DCHP was site specific based on their hospital population, organisational support and resources. Our study also demonstrated an improvement in nonclinical staff-perceived level of job satisfaction in working with patients with CI. These findings are important as both clinical and non-clinical staff job satisfaction is positively related to patient satisfaction and the quality of care provided in an acute hospital setting $[14,21]$.

Within the hospital environment, the provision of highquality care for patients with CI requires organisational support. While the involvement of clinical and non-clinical staff is important for driving cultural change, high-level

Table 5 Staff reporting difficulty when working with patients and carers: by clinical status

\begin{tabular}{llll}
\hline Self-rated measures & Pre-Intervention & $\begin{array}{l}\text { Post- } \\
\text { Intervention }\end{array}$ \\
& $\frac{\mathrm{n}}{\mathrm{n}} \%$ & $P$ \\
\hline
\end{tabular}

Difficulty working with patients with dementia, delirium or memory and thinking difficulties

$\begin{array}{llllll}\text { Clinical staff } & 722 & 89.6 & 318 & 80.6 & 0.001^{* *} \\ \text { Non clinical staff } & 144 & 28.4 & 26 & 46.7 & 0.220 \\ \text { All staff } & 944 & 82.2 & 418 & 78.5 & 0.220\end{array}$

Difficulty working with the carer or family of patients with dementia, delirium or memory and thinking difficulties

\begin{tabular}{llllll} 
Clinical staff & 720 & 65.9 & 317 & 49.1 & $<0.001^{* *}$ \\
Non clinical staff & 142 & 15.4 & 26 & 7.1 & 0.680 \\
All staff & 939 & 58.4 & 415 & 47.8 & $0.004^{*}$ \\
\hline
\end{tabular}

*Significant at the 0.05 level

** Significant at the 0.001 level 
organisational commitment and support is essential for sustaining and supporting this change [22]. In particular, hospitals with a negative culture of care, labelling people with dementia as 'difficult' have been found to negatively influence the well-being of people with dementia [23, 24]. Following the implementation of the DCHP, staff perceptions of the organisational support they received from the hospital improved overall. This is consistent with the literature which has highlighted the significant impact that the organisational and psychosocial working conditions, have on the health and well-being of people with CI [25]. Edvardsson et al. [25] provided recommendations for modifying an acute hospital environment, in order to better meet the needs of the older patients with CI. It was suggested that, adjusting the hospital environment by providing a balanced approach to care, which includes the provision of core knowledge and skills for all staff, and access to CI expertise in acute hospitals. This suggests that in order for staff training to affect the acute care outcomes for patients with CI, organisational change within the hospital environment is necessary [26].

\section{Limitations}

One of the limitations of this study was that the fidelity of the DCHP education implementation was not assessed and may have impacted the outcomes. However, this is a pragmatic study and involved implementing the DCHP education within the constraints of a real clinical environment, respecting that differences existed between the participating hospitals as a result of their hospitalised population, practice differences, data coding differences and organisational resources. An additional limitation is that the sustainability of the education program was not measured. However, this is an important measure to examine and should be the basis of further research. The post-intervention survey responses were lower than the pre-intervention survey responses. This was because the pre-intervention survey was distributed during planned DCHP training sessions in the hospitals. The post-intervention surveys were distributed to staff on the wards, up to 6 months post intervention, and it is likely that the timing and distribution method impacted on the response rate. Psychometrics were not completed for the survey used in this study and this is an acknowledged limitation.

\section{Conclusions}

Overall, staff reported increased confidence, comfort and job satisfaction in caring for patients with CI, following the implementation of the DCHP education program. The overall positive results around staff satisfaction have significant implications for the improvement of care for patients with $\mathrm{CI}$, as well as staff retention and job satisfaction. Further research is required in order to determine whether these improvements are sustained in the long term.

\section{Additional files}

Additional file 1: Cognitive Impairment Identifier (CII). The $\mathrm{Cll}$ bedside alert is a copyright product of Ballarat Health Services. Patients who screen positive for $\mathrm{Cl}$ are offered placement of the $\mathrm{Cll}$ above their bedside. The CII is a key component of the DCHP and its visibility enables all hospital staff (clinical and non-clinical) to assist patients with $\mathrm{Cl}$. (PDF $139 \mathrm{~kb}$ )

Additional file 2: Dementia Care in Hospitals Program Key Communication Strategies. The nine key communication strategies used as part of the DCHP educational training program. (PDF 137 kb)

Additional file 3: Pre-Intervention Staff Satisfaction Survey. The staff satisfaction survey completed by staff prior to implementation of the DCHP educational training program. (PDF $185 \mathrm{~kb}$ )

Additional file 4: Post-Intervention Staff Satisfaction Survey. The staff satisfaction survey completed by staff after implementation of the DCHP educational training program. (PDF $186 \mathrm{~kb}$ )

\section{Abbreviations}

Cl: Cognitive impairment; Cll: Cognitive Impairment Identifier; DCHP: Dementia Care in Hospitals Program

\section{Acknowledgements}

The Ballarat Health Services team acknowledges the financial support of Commonwealth Government of Australia grant no. 4-3VTSOC to undertake the National Rollout of the Dementia Care in Hospitals Program (DCHP). Thanks to the National Stakeholders Advisory Group (NSAG) for their guidance and input over the course of the project. Thanks to members of the Deakin University evaluation team, the Evaluation Advisory Group. Ballarat Health Services also acknowledges the support of Health Roundtable (HRT)

Ballarat Health Services would like to thank Alzheimer's Australia for the ongoing support and endorsement of the DCHP and the Cognitive Impairment Identifier.

Ballarat Health Services also acknowledges the work, energy, and sustained commitment of our project partners in driving culture change in the care of patients with cognitive impairment in the acute setting.

\section{Authors' contributions}

MY, MT, MM contributed to the conception and design of the study. MEM, AWS, EW, MM, MT, W and MY contributed to the acquisition, analysis, and interpretation of data; drafting the article; and revising it critically for important academic content. All authors approved the final version for publication.

\section{Funding}

The Ballarat Health Services team received funding from the Commonwealth Government of Australia (grant no. 4-3VTSOC) to undertake the National Rollout of the Dementia Care in Hospitals Program (DCHP). This funding body played no active role in the design of the study or collection, analysis, interpretation of data or writing of this manuscript. This grant funded EFT 0.2 for three members of the research team to manage the National Roll-out. Deakin University, School of Health and Social Development were funded by the grant to run the rollout evaluation.

\section{Availability of data and materials}

The datasets used and analysed during the current study available from the corresponding author on reasonable request.

\section{Ethics approval and consent to participate}

Written informed consent was obtained from all participants. The study was granted ethics approval from the relevant Human Research Ethics

Committee at each participating site. Ethics approval numbers are:

1. HREC/15/TQEH/9 (Government of South Australia, SA Health, and Human Research Ethics Committee); 
2. ETH.6.15.105 (ACT Health, Human Research Ethics Committee); 3. HREC/15/TQEH/9 (Human Research Ethics Committee (Tasmania) Network) 4. 2015-103 (Government of Western Australia, Department of Health, Human Research Ethics Committee).

\section{Consent for publication}

Written informed consent was obtained from all participants for publication of their data.

\section{Competing interests}

The authors declare that they have no competing interests

\section{Author details}

'Deakin University, Geelong Australia, Deakin Rural Health, School of Medicine, Warrnambool, VIC 3280, Australia. ${ }^{2}$ Ballarat Health Services (BHS), Ballarat 3350, Australia. ${ }^{3}$ Deakin University, Geelong Australia, Faculty of Health, School of Medicine, Geelong 3220, Australia.

Received: 29 October 2018 Accepted: 28 August 2019

Published online: 18 September 2019

\section{References}

1. Australian Institute of Health and Welfare. Dementia in Australia. Canberra: AlHW; 2012

2. Reynish EL, Hapca SM, De Souza N, Cvoro V, Donnan PT, Guthrie B. Epidemiology and outcomes of people with dementia, delirium, and unspecified cognitive impairment in the general hospital: prospective cohort study of 10,014 admissions. BMC Med. 2017;15(1):140.

3. Alzheimer's Australia. Economic cost of dementia in Australia: 2016-2056. Canberra: Alzheimer's Australia; 2017.

4. Australian Commission on Safety and Quality in Health Care. National safety and quality health service standards. Sydney: ACSQHC; 2017.

5. Australian Commission on Safety and Quality in Health Care. A better way to care: safe and high-quality care for patients with cognitive impairment (dementia and delirium) in hospital - actions for clinicians. Sydney: ACSQHC; 2014.

6. Clissett P, Porock D, Harwood RH, Gladman JR. The challenges of achieving person-centred care in acute hospitals: a qualitative study of people with dementia and their families. Int J Nurs Stud. 2013;50(11):1495-503.

7. Schindel ML, Gillies L, Coker E, Pizzacalla A, Montemuro M, Suva G, et al. An education intervention to enhance staff self-efficacy to provide dementia care in an acute care hospital in Canada: a nonrandomized controlled study. Am J Alzheimers Dis Other Dement. 2016;31(8):664-77.

8. Australian Commission on Safety and Quality in Health Care. Evidence for the safety and quality issues associated with the care of patients with cognitive impairment in acute settings: a rapid review. Sydney: ACSQHC; 2013.

9. Albinsson L, Strang P. Existential concerns of families of late-stage dementia patients: questions of freedom, choices, isolation, death, and meaning. J Palliat Med. 2003;6(2):225-35

10. Doyle C. International perspectives on dementia education, training and knowledge transfer. Int Psychogeriatr. 2009;21(Suppl 1):S1-2.

11. Robison J, Pillemer K. Job satisfaction and intention to quit among nursing home nursing staff: do special care units make a difference? J Appl Gerontol. 2007:26(1):95-112.

12. Nemmaniwar A, Deshpande M. Job satisfaction among hospital employees: a review of literature. IOSR J Bus Manag. 2016;18(6):27-31.

13. Foreman P, Gardner I. Evaluation of education and training of staff in dementia care and Management in Acute Settings. Bundoora: Australian Institute for Primary Care; 2007

14. Vernooij-Dasssen MJ, Faber MJ, Olde Rikkert MG, Koopmans RT, van Achterberg T, Braat DD, et al. Dementia care and labour market: the role of job satisfaction. Aging Ment Health. 2009;13(3):383-90.

15. Surr CA, Smith SJ, Crossland J, Robins J. Impact of a person-centred dementia care training programme on hospital staff attitudes, role efficacy and perceptions of caring for people with dementia: a repeated measures study. Int J Nurs Stud. 2016;53:144-51.

16. Yates M, Watts JJ, Bail K, Mohebbi M, MacDermott S, Jebramek J, Brodaty H. Evaluating the impact of the Dementia Care in Hospitals Program (DCHP) on hospital-acquired complications: study protocol. Int J Environ Res Public Health. 2018;15(9):1878.
17. Brown JA, Sait K, Faine R, Huskins L. Service and support requirements of people with younger onset dementia and their families. Sydney: Department of Family and Community Services, Ageing, Disability and Home Care; 2012

18. Yarber L, Brownson CA, Jacob RR, Baker EA, Jones E, Baumann C, et al. Evaluating a train-the-trainer approach for improving capacity for evidence-based decision making in public health. BMC Health Serv Res. 2015;15:547.

19. Hughes J, Bagley H, Reilly S, Burns A, Challis D. Care staff working with people with dementia: training, knowledge and confidence. Dementia. 2008:7(2):227-38.

20. Sampson EL, Vickerstaff $V$, Lietz S, Orrell M. Improving the care of people with dementia in general hospitals: evaluation of a whole-system train-the-trainer model. Int Psychogeriatr. 2017;29(4):605-14.

21. Chater K, Hughes N. Strategies to deliver dementia training and education in the acute hospital setting. J Res Nurs. 2013;18(6):578-93.

22. Schall M, Sevin C, Wasson JH. Making high-quality, patient-centered care a reality. J Ambul Care Manage. 2009;32(1):3-7.

23. Cowdell $F$. The care of older people with dementia in acute hospitals. Int J Older People Nursing. 2010;5(2):83-92.

24. National Audit Office. Improving dementia services in England - an interim report. London: National Audit Office; 2010.

25. Edvardsson D, Nay R. Acute care and older people: challenges and ways forward. Aust J Adv Nurs. 2010:27(2):63-9.

26. Moyle W, Murfield JE, Griffiths SG, Venturato L. Assessing quality of life of older people with dementia: a comparison of quantitative self-report and proxy accounts. J Adv Nurs. 2012;68(10):2237-46.

\section{Publisher's Note}

Springer Nature remains neutral with regard to jurisdictional claims in published maps and institutional affiliations.

Ready to submit your research? Choose BMC and benefit from:

- fast, convenient online submission

- thorough peer review by experienced researchers in your field

- rapid publication on acceptance

- support for research data, including large and complex data types

- gold Open Access which fosters wider collaboration and increased citations

- maximum visibility for your research: over $100 \mathrm{M}$ website views per year

At $\mathrm{BMC}$, research is always in progress.

Learn more biomedcentral.com/submissions 